\title{
Novel Rickettsia Species Infecting Dogs, United States
}

\author{
James M. Wilson, Edward B. Breitschwerdt, Nicholas B. Juhasz, Henry S. Marr,
} Joao Felipe de Brito Galvão, Carmela L. Pratt, Barbara A. Qurollo

In 2018 and 2019, spotted fever was suspected in 3 dogs in 3 US states. The dogs had fever and hematological abnormalities; blood samples were Rickettsia seroreactive. Identical Rickettsia DNA sequences were amplified from the samples. Multilocus phylogenetic analysis showed the dogs were infected with a novel Rickettsia species related to human Rickettsia pathogens.

Tn the United States, tickborne Rickettsia parkeri, $R$. 1 philipii (Rickettsia 364D), and R. rickettsii, causative agents of Rocky Mountain spotted fever (RMSF), are well-documented human spotted fever group (SFG) rickettsioses (1). $R$. rickettsii is the only known cause of SFG rickettsioses in dogs (2). The extent to which other SFG Rickettsia are pathogenic in dogs is unclear; however, SFG Rickettsia seroprevalence is high among dogs in the United States and Mexico $(3,4)$. The increased $R$. rickettsii seroprevalence in humans in the United States during the past decade has been attributed to SFG Rickettsia cross-reactivity $(1,5)$.

We report 3 dogs with febrile illness located in different US states. Samples from the dogs were $R$. rickettsii seroreactive. Identical Rickettsia DNA gene sequences were obtained from each dog's blood specimen and used to investigate Rickettsia spp.

\section{The Cases}

On May 15, 2018, a 10-year-old male neutered mixed breed dog (case 1) from Tennessee was examined by a veterinarian for lethargy and hyporexia. The owner reported removing a tick (species unknown) within the previous 2 weeks. On physical examination, the dog had fever $\left(39.8^{\circ} \mathrm{C}\right)$ and possible hepatomegaly.

\footnotetext{
Author affiliations: North Carolina State University College of Veterinary Medicine, Raleigh, NC, USA (J.M. Wilson,

E.B. Breitschwerdt, N.B. Juhasz, H.S. Marr, B.A. Qurollo); VCA Arboretum View Animal Hospital, Downers Grove, Illinois, USA (J.F. de Brito Galvão); Oklahoma Veterinary Specialists, Tulsa Oklahoma, USA (C.L. Pratt)
}

DOI: https://doi.org/10.3201/eid2612.200272
Radiographic imaging results were unremarkable. Thrombocytopenia was the only abnormality noted on complete blood count (CBC). Serum biochemistry panel (SBP) abnormalities included hyperglobulinemia, increased serum alkaline phosphatase activity, hypoglycemia, and hyponatremia (Table 1). Results of urine dipstick and sediment examination were unremarkable. The dog's samples were $R$. rickettsii seroreactive and PCR positive for Rickettsia (Table 2). Clinical abnormalities resolved after treatment with doxycycline, and the dog remained healthy during the 1-year follow-up period.

On May 8, 2019, a 9-year-old male neutered Boston terrier (case 2) from Illinois was examined by a veterinarian for lethargy, difficulty walking, and painful elbows. Clinical signs developed 3 days after returning from a tick-infested area in Arkansas. Abnormalities noted on physical examination included fever $\left(40.1^{\circ} \mathrm{C}\right)$, dehydration, joint effusion, elbow pain, and shifting leg lameness. Thrombocytopenia and mild leukocytosis were the only CBC abnormalities (Table 1). SBP abnormalities included hypoalbuminemia, increased alanine amino transferase activity, alkaline phosphatase activity, hypercholesterolemia, and hypocalcemia (Table 1). Mild microalbuminuria was noted. Neutrophilic inflammation was documented by synovial fluid cytology in the right and left stifle joints, right tarsus, and left elbow joint. The left carpus contained moderate, chronic inflammation with very rare extracellular cocci; however, culture resulted in no bacterial growth. The dog experienced cardiorespiratory arrest during sedated arthrocentesis but recovered after CPR and sedative reversal. Thoracic radiographs were unremarkable. The dog's samples were $R$. rickettsii seroreactive and PCR-positive for Rickettsia and convalescent titers demonstrated 4-fold seroconversion (Table 2). Most clinical abnormalities resolved after administration of doxycycline to treat rickettsiosis, prednisone to treat potential immune-mediated component, omeprazole to prevent gastric ulcers, and metronidazole to treat 
Table 1. Findings from physical examination, laboratory results, treatment regimens for 3 dogs infected with a novel Rickettsia species, United States*

\begin{tabular}{lc}
\hline $\begin{array}{l}\text { Examination } \\
\text { and treatment }\end{array}$ & Case 1 \\
\hline $\begin{array}{l}\text { Physical } \\
\text { examination }\end{array}$ & Febrile $\left(39.8^{\circ} \mathrm{C}\right)$; lethargy; \\
$+/-$ hepatomegaly
\end{tabular}

CBC Platelets $141 \times 10^{3} \mathrm{cells} / \mu \mathrm{L}$ (RI $200-500 \times 10^{3}$ cells $\left./ \mu \mathrm{L}\right)$
Case 2

Febrile $\left(40.1^{\circ} \mathrm{C}\right)$; lethargy; dehydration; joint effusion (elbow, carpus, and tarsus); arthropathy; shifting leg lameness Platelets $139 \times 10^{3}$ cells $/ \mu \mathrm{L}$ (RI $170-400 \times 10^{3}$ cells $/ \mu \mathrm{L}$ )
Case 3

Febrile $\left(39.8^{\circ} \mathrm{C}\right)$; lethargy; abdominal pain
Platelets $60 \times 10^{3}$ cells $/ \mu \mathrm{L}$ (RI 125-

$500 \times 10^{3}$ cells $/ \mu \mathrm{L}$ ); Hct $35.2 \%$ (RI

$36 \%-55 \%$ ); 2 d later platelets $25 \times$ $10^{3}$ cells $/ \mu \mathrm{L}$ and Hct $26.8 \%$

\begin{tabular}{|c|c|c|c|}
\hline SBP & $\begin{array}{l}\text { Globulins } 4.5 \mathrm{~g} / \mathrm{dL}(\mathrm{RI} 2.1-4.4 \mathrm{~g} / \mathrm{dL}) \\
\text { ALP } 177 \mathrm{U} / \mathrm{L}(\mathrm{RI} 11-140 \mathrm{U} / \mathrm{L}) ; \\
\text { glucose } 73 \mathrm{mg} / \mathrm{dL}(\mathrm{RI} 75-125 \\
\mathrm{mg} / \mathrm{dL}) ; \text { sodium } 136.5 \mathrm{mmol} / \mathrm{L}\end{array}$ & $\begin{array}{c}\text { Albumin } 2.2 \text { g/dL (RI 2.7-4.4 g/dL); ALT } \\
\text { 1,158 U/L (RI 12-118 U/L); ALP 1,702 } \\
\text { U/L (RI 5-131 U/L); cholesterol } 352 \\
\text { mg/dL (RI 92-324 mg/dL); calcium } 8.4\end{array}$ & $\begin{array}{c}\text { Albumin } 1.0 \mathrm{mg} / \mathrm{dL}(\mathrm{RI} 2.5-4.3 \\
\mathrm{mg} / \mathrm{dL}) ; \text { calcium } 8.4 \mathrm{mg} / \mathrm{dL} \\
\text { (RI 8.9-11.4 mg/dL); BUN } 35 \\
\text { (35, RI } 7-28 \mathrm{mg} / \mathrm{dL})\end{array}$ \\
\hline
\end{tabular}
(RI $143-153 \mathrm{mmol} / \mathrm{L}$ )

\begin{tabular}{lc}
\hline Urinalysis & USG 1.007 \\
\hline $\begin{array}{l}\text { Treatment } \\
\text { regimen }\end{array}$ & Doxycycline $(6 \mathrm{mg} / \mathrm{kg}$ every \\
$12 \mathrm{~h}$ for $21 \mathrm{~d})$
\end{tabular}
$\mathrm{mg} / \mathrm{dL}$ (RI 8.9-11.4 mg/dL) Microalbuminuria $3.1(\mathrm{Rl}<2.5 \mathrm{mg} / \mathrm{dL})$

\section{Doxycycline $(7 \mathrm{mg} / \mathrm{kg}$ every $12 \mathrm{~h}$ for 28} d); prednisone $(1 \mathrm{mg} / \mathrm{kg}$ every $12 \mathrm{~h}$ for 9 mo with gradual taper); omeprazole

$(1.4 \mathrm{mg} / \mathrm{kg}$ every $12 \mathrm{~h}$ for $9 \mathrm{mo}$ ); ondansetron $(0.5 \mathrm{mg} / \mathrm{kg}$ every $12 \mathrm{~h}$ for $15 \mathrm{~d}$ ); and metronidazole $(17 \mathrm{mg} / \mathrm{kg}$ every $12 \mathrm{~h}$ for $15 \mathrm{~d})$
USG 1.033; 3+ proteinuria; UPC 14.7 (RI $0.00-1.00)$

Doxycycline $(7.5 \mathrm{mg} / \mathrm{kg} \mathrm{q} 12 \mathrm{~h}$ for $40 \mathrm{~d}$ ); prednisone (1 $\mathrm{mg} / \mathrm{kg}$ every $12 \mathrm{~h}$ for $14 \mathrm{~d}$, then $0.5 \mathrm{mg} / \mathrm{kg}$ every $12 \mathrm{~h}$ for $6 \mathrm{~d}$, then every $24 \mathrm{~h}$ for 22 $\mathrm{d}$ until death), mycophenolate

$(12.5 \mathrm{mg} / \mathrm{kg}$ every $12 \mathrm{~h}$ for 22 d until death)

${ }^{*} \mathrm{ALP}$, alkaline phosphatase activity; ALT, alanine amino transferase activity; BUN, blood urea nitrogen; CBC, complete blood count; Hct, hematocrit; RI, reference interval; SBP, serum biochemistry panel; UA, urinalysis; UPC, urine protein/creatinine ratio; USG, urine specific gravity.

assumed dysbiosis. All SBP changes resolved within 5 months of treatment and the dog remained healthy during the 5-month follow-up.

On August 28, 2019, a 9-year-old male neutered terrier mixed-breed (case 3) from Oklahoma was examined by a veterinarian for lethargy, hyporexia, and polydipsia. Physical examination revealed fever $\left(39.8^{\circ} \mathrm{C}\right)$ and palpable abdominal tenderness. CBC abnormalities included a normocytic normochromic anemia and thrombocytopenia. SBP abnormalities included hypoproteinemia, hypocalcemia, and mild azotemia. A protein-losing nephropathy (PLN) was documented by urine dipstick and protein/creatinine ratio. Blood samples were $R$. rickettsii seroreactive and Rickettsia PCR positive, and convalescent titers demonstrated 4-fold seroconversion (Table 2).

\begin{tabular}{|c|c|c|c|c|c|c|c|c|c|c|c|}
\hline \multirow[b]{3}{*}{ Sample dates } & \multirow{3}{*}{$\begin{array}{l}\text { CVBD } \\
\text { panel† }\end{array}$} & \multirow[b]{3}{*}{ IFA‡ } & \multicolumn{9}{|c|}{ Rickettsia PCR§ } \\
\hline & & & \multirow{2}{*}{$\begin{array}{c}23 S-5 S \\
\text { ITS }\end{array}$} & \multirow{2}{*}{$\begin{array}{c}\text { htrA } \\
(17 \mathrm{kDa})\end{array}$} & \multirow{2}{*}{$\begin{array}{c}\text { mmpA-purC } \\
\text { ITS }\end{array}$} & \multicolumn{3}{|c|}{ gltA region } & \multicolumn{3}{|c|}{ ompA region } \\
\hline & & & & & & 1 & 2 & 3 & 1 & 2 & 3 \\
\hline \multicolumn{12}{|l|}{ Case 1} \\
\hline 2018 May 5ा & - & $1: 512$ & + & + & + & + & + & + & + & + & $-{ }^{* *}$ \\
\hline \multicolumn{12}{|l|}{ Case 2} \\
\hline 2019 May 8ा & - & $1: 256$ & + & + & + & + & NAt† & + & + & + & NAt† \\
\hline 2019 May 15 & - & $1: 8,192$ & - & NA & NA & NA & NA & NA & NA & NA & NA \\
\hline 2019 May 28 & - & $1: 1,024$ & - & NA & NA & NA & NA & NA & NA & NA & NA \\
\hline 2019 Jul 16 & NA & $1: 2,048$ & NA & NA & NA & NA & NA & NA & NA & NA & NA \\
\hline 2019 Oct 2 & - & $1: 2,048$ & - & NA & NA & NA & NA & NA & NA & NA & NA \\
\hline 2019 Nov 12 & - & $1: 2,048$ & - & NA & NA & NA & NA & NA & NA & NA & NA \\
\hline \multicolumn{12}{|l|}{ Case 3} \\
\hline 2019 Aug 28㧊 & - & $1: 1,024$ & + & + & + & + & + & + & + & + & + \\
\hline 2019 Sep 10 & NA & $1: 8,192$ & - & NA & NA & NA & NA & NA & NA & NA & NA \\
\hline \multicolumn{12}{|c|}{ 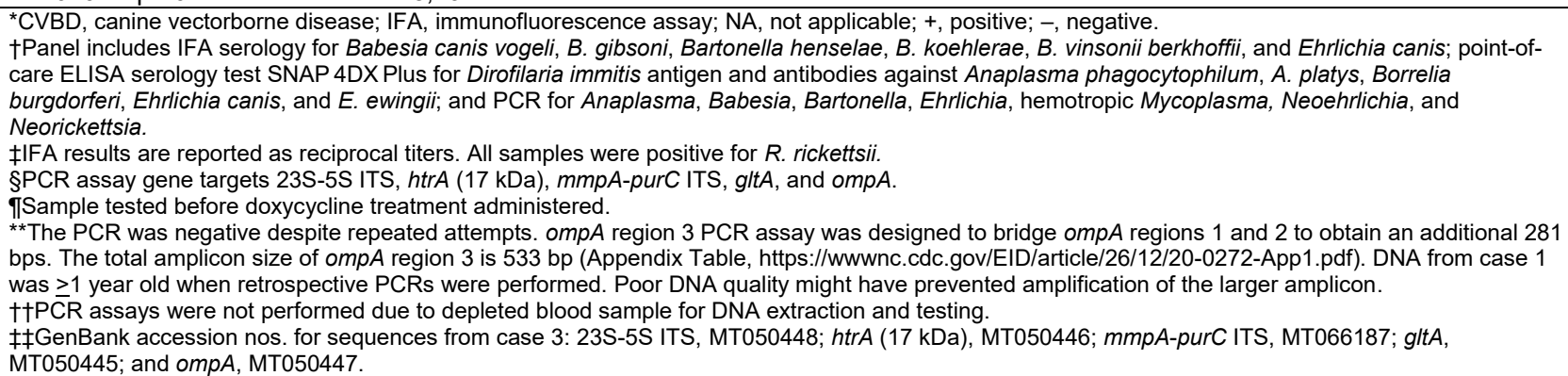 } \\
\hline
\end{tabular}


The year before, in August 2018, the dog described in case 3 was examined by a veterinarian for lethargy after tick attachment. At that time, fever $\left(39.7^{\circ} \mathrm{C}\right)$, anemia, thrombocytopenia, hyperbilirubinemia, and hypoproteinemia were documented. IFA serology tests performed by 1 diagnostic laboratory showed samples were $R$. rickettsii seroreactive (1:320) but seronegative for Anplasma spp., Borrelia burdorferi, and Ehrlichia spp. by SNAP 4Dx Plus (IDEXX Laboratories, https://www.idexx.com). Doxycycline and immunosuppressive doses of prednisone were administered concurrently for RMSF and potential immune-mediated disease. Clinical and hematologic abnormalities resolved, and treatment was transitioned from prednisone to cyclosporine due to adverse side effects. Cyclosporine was discontinued in January 2019 and serial monthly CBCs remained normal through March 2019. When rechecked on August 9, 2019, for joint pain, hematocrit and platelet count were normal, but hypoproteinemia, hypoalbuminemia, and hypocalcemia were detected. By August 30, 2019, the dog's anemia and thrombocytopenia worsened, despite treatment with doxycycline and prednisone. Marked abdominal effusion was documented by abdominal ultrasound, without evidence of an intra-abdominal mass. Prednisone and mycophenolate were administered for presumptive immune-mediated thrombocytopenia, and within 3 weeks, the platelet count normalized and titers increased by 4 -fold. Despite medical therapy for PLN, nephrotic syndrome developed, and the dog was euthanized.

We obtained identical Rickettsia DNA gene sequences from each dog's blood specimen. We confirmed novel Rickettsia sp. by PCR targeting 3 genes (gltA, htrA, and ompA) and 2 intergenic spacer re-

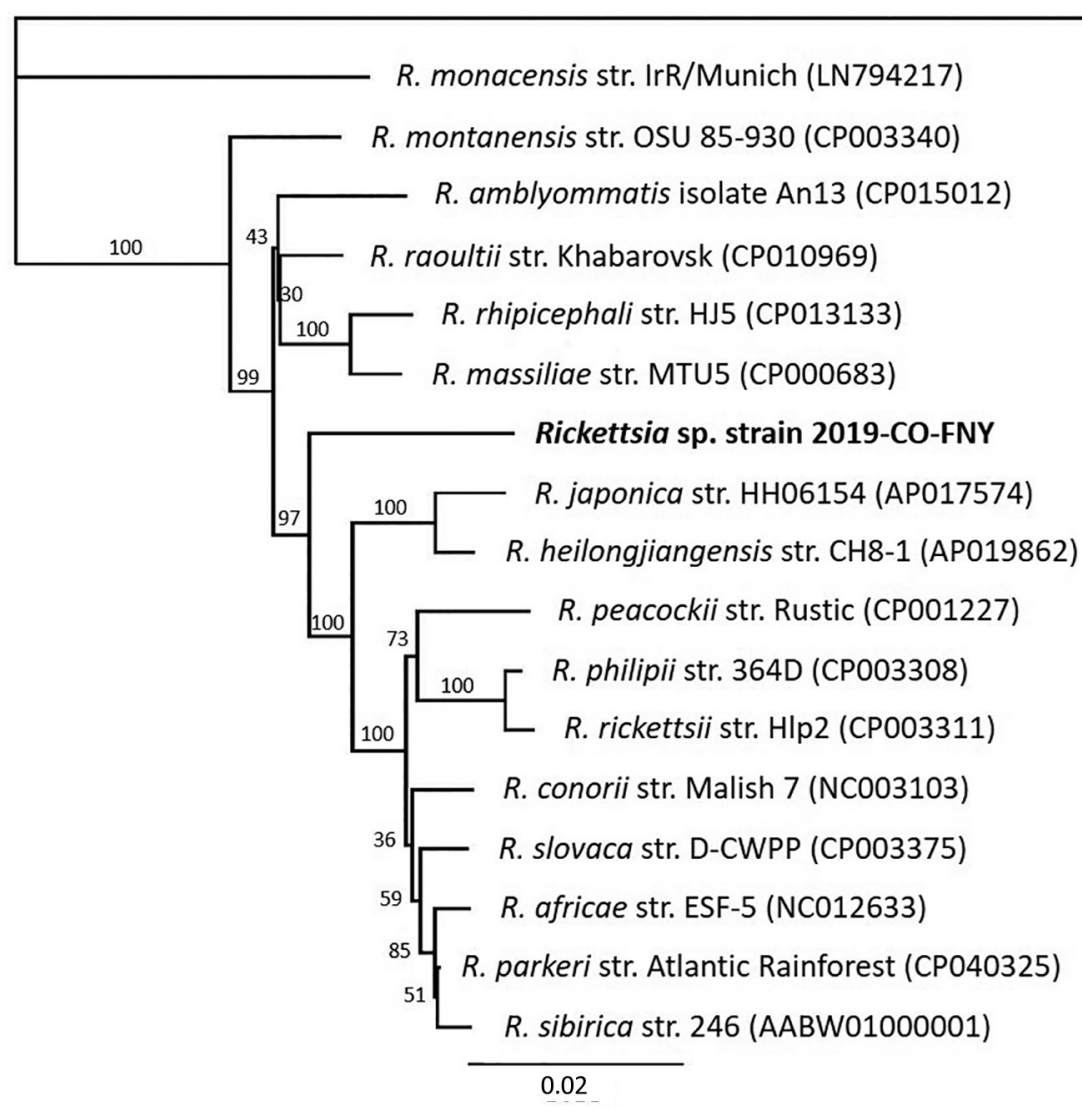

R. canadensis str. Mckiel (CP000409)

Figure. Multilocus phylogenetic tree of Rickettsia spp. obtained from a dog with Rocky Mountain spotted fever-type symptoms in 2019 (bold) compared with reference sequences. We noted 3 dogs with RSMF symptoms. Rickettsia DNA were identical among all 3 cases; however, complete sequences from all 5 regions were obtained only from case 3 , which we used to represent the novel Rickettsia species strain 2019-CO-FNY. We used 2,576 nucleotides concatenated from regions within 3 genes ( $g$ lt $A$, $h$ trA, and ompA) and 2 intergenic spacer regions (23S-5S and $m m p A-p u r C$ ). We used the maximum-likelihood method and Tamura-Nei model $(6,7)$ optimized for branch length, topology, and substitution rate to assemble the tree by using the PhyML 3.3.20180621 plugin in Geneious Prime 11.0.0+7 (https:// www.geneious.com). Numbers at nodes indicate bootstrap percentages obtained from 1,000 resamplings. Numbers in parentheses are GenBank accession numbers. The tree is drawn to scale. Scale bar indicated the number of nucleotide substitutions per site. 
gions (23S-5S and mmpA-purC) (Table 2). Rickettsia amplicons were $100 \%$ identical among the 3 dogs. We amplified a larger region of the ompA and gltA genes by using 3 different quantitative PCRs from case 3 . We submitted sequences from this dog's serum samples to GenBank (accession nos. MT050445-8 and MT066187). We also used the Rickettsia sequences from case 3 to generate a phylogenetic tree (Table 2) based on concatenated novel Rickettsia sp. DNA sequences and reference Rickettsia spp. We generated the phylogenetic tree by using the maximum-likelihood method based on the Tamura-Nei model (Figure) $(6,7)$. Multilocus phylogenetic analysis placed the novel Rickettsia sp. in a clade among SFG Rickettsia between the human pathogens $R$. heilongjiangensis and $R$. massiliae. We attempted cell culture isolation of the Rickettsia sp. from whole blood but were unsuccessful (Appendix, https://wwwnc.cdc.gov/EID/ article/26/12/20-0272-App1.pdf).

\section{Conclusions}

We report similar illnesses among 3 dogs from different US states associated with tick exposures occurring in summer months. All 3 cases demonstrated fever, lethargy, and thrombocytopenia, abnormalities commonly associated with RMSF. Case 1 had a typical acute onset fever and rapidly responded to treatment with doxycycline; case 2 had a neutrophilic polyarthritis, which has been associated with RMSF in dogs (8). Case 3 was examined for acute onset febrile illness 1 year before the novel Rickettsia sp. infection was documented; Rickettsia IFA seroreactivity was documented on both occasions. This dog likely had an unidentified, concurrent disease process that contributed to PLN.

The cases were geographically distributed among 4 states; the dogs resided in Illinois, Oklahoma, and Tennessee, but the dog from Illinois had traveled to a tick-infested area of Arkansas. The tick species were not identified, but ticks common to these states include Amblyomma americanum, Dermacentor variabilis, and Rhipicephalus sanguineus sensu lato, all of which are known to transmit Rickettsia (3). Haemophysalis longicornis, an invasive tick species recently confirmed in the United States, including in Tennessee and Arkansas, should be considered a potential vector for Rickettsia spp. $(9,10)$.

Based on serologic cross-reactivity, presence of $\operatorname{ompA}$, and phylogenetic tree analysis, the new Rickettsia sp. is an SFG Rickettsia, phylogenetically related to human pathogenic $R$. heilongjiangensis and R. massiliae, with only $95 \%$ identity to each $(11,12)$. Thus, we report a previously unknown and unique
Rickettsia sp. with clinical significance for dogs and potentially humans. Because this novel Rickettsia cross-reacts with $R$. rickettsia on IFA, it could be underdiagnosed and more geographically widespread. Studies aimed at identifying the tick vector, potential animal reservoirs, and prevalence are ongoing. These 3 canine rickettsioses cases underscore the value of dogs as sentinels for emerging tickborne pathogens $(13,14)$.

\section{Acknowledgments}

We thank Brad L. Fry for providing the clinical and diagnostic information for case 1 in this manuscript.

B.A.Q. is co-director of the North Carolina State Vector Borne Disease Diagnostic Lab (NC State VBDDL); IDEXX Laboratories (https://www.idexx.com) funds a portion of her salary. E.B.B. codirects the NC State VBDDL and the Intracellular Pathogens Research Laboratory at NC State, is chief scientific officer at Galaxy Diagnostics (https://www.galaxydx.com), and is a paid consultant for IDEXX Laboratories, Inc. No other authors have competing interests to declare.

\section{About the Author}

Mr. Wilson is a vectorborne disease research technician at the North Carolina State Vector Borne Disease Diagnostic Lab. His research interests include optimization of molecular testing and vectorborne infectious disease diagnoses.

\section{References}

1. Straily A, Stuck S, Singleton J, Brennan S, Marcum S, Condit M, et al. Antibody titers reactive with Rickettsia rickettsii in blood donors and implications for surveillance of spotted fever Rickettsiosis in the United States. J Infect Dis. 2020;221:1371-8. https://doi.org/10.1093/infdis/jiz316

2. Warner RD, Marsh WW. Rocky Mountain spotted fever. J Am Vet Med Assoc. 2002;221:1413-7. https://doi.org/10.2460/javma.2002.221.1413

3. Hardstone Yoshimizu M, Billeter SA. Suspected and confirmed vector-borne rickettsioses of North America associated with human diseases. Trop Med Infect Dis. 2018;3:2. https://doi.org/10.3390/tropicalmed3010002

4. Yancey CB, Hegarty BC, Qurollo BA, Levy MG, Birkenheuer AJ, Weber DJ, et al. Regional seroreactivity and vector-borne disease co-exposures in dogs in the United States from 20042010: utility of canine surveillance. Vector Borne Zoonotic Dis. 2014;14:724-32. https://doi.org/10.1089/vbz.2014.1592

5. Drexler NA, Dahlgren FS, Heitman KN, Massung RF, Paddock CD, Behravesh CB. National surveillance of spotted fever group rickettsioses in the United States, 2008-2012. Am J Trop Med Hyg. 2016;94:26-34. https://doi.org/10.4269/ ajtmh.15-0472

6. Tamura K, Nei M. Estimation of the number of nucleotide substitutions in the control region of mitochondrial DNA in humans and chimpanzees. Mol Biol Evol. 1993;10:512-26. https://doi.org/10.1093/oxfordjournals.molbev.a040023

7. Guindon S, Dufayard JF, Lefort V, Anisimova M, 
Hordijk W, Gascuel O. New algorithms and methods to estimate maximum-likelihood phylogenies: assessing the performance of PhyML 3.0. Syst Biol. 2010;59:307-21. https://doi.org/10.1093/sysbio/syq010

8. Gasser AM, Birkenheuer AJ, Breitschwerdt EB. Canine Rocky Mountain spotted fever: a retrospective study of 30 cases. J Am Anim Hosp Assoc. 2001;37:41-8. https://doi.org/ 10.5326/15473317-37-1-41

9. Raghavan RK, Barker SC, Cobos ME, Barker D, Teo EJM, Foley $\mathrm{DH}$, et al. Potential spatial distribution of the newly introduced long-horned tick, Haemaphysalis longicornis in North America. Sci Rep. 2019;9:498. https:/ /doi.org/ 10.1038/s41598-018-37205-2

10. Xu H, Zhang Q, Guan H, Zhong Y, Jiang F, Chen Z, et al. High incidence of a novel Rickettsia genotype in parasitic Haemaphysalis longicornis from China-North Korea Border. Sci Rep. 2019;9:5373. https:/ / doi.org/10.1038/ s41598-019-41879-7

11. Ando S, Kurosawa M, Sakata A, Fujita H, Sakai K, Sekine M, et al. Human Rickettsia heilongjiangensis infection, Japan. Emerg Infect Dis. 2010;16:1306-8. https://doi.org/10.3201/eid1608.100049

12. Vitale G, Mansuelo S, Rolain J-M, Raoult D. Rickettsia massiliae human isolation. Emerg Infect Dis. 2006;12:174-5. https://doi.org/10.3201/eid1201.050850

13. Estrada I, Balagot C, Fierro M, Kriner P, Iniguez-Stevens E, Kjemtrup A, et al. Spotted fever group rickettsiae canine serosurveillance near the US-Mexico border in California. Zoonoses Public Health. 2020;67:148-55. https:/ / doi.org/ 10.1111/zph.12666

14. Smith FD, Ballantyne R, Morgan ER, Wall R. Estimating Lyme disease risk using pet dogs as sentinels. Comp Immunol Microbiol Infect Dis. 2012;35:163-7. https:/ / doi.org/ 10.1016/j.cimid.2011.12.009

Address for correspondence: Barbara A. Qurollo, Department of Clinical Sciences College of Veterinary Medicine, North Carolina State University, Research Bldg, Office 464, 1060 William Moore Dr, Raleigh, NC 27606, USA; email: baquroll@ncsu.edu

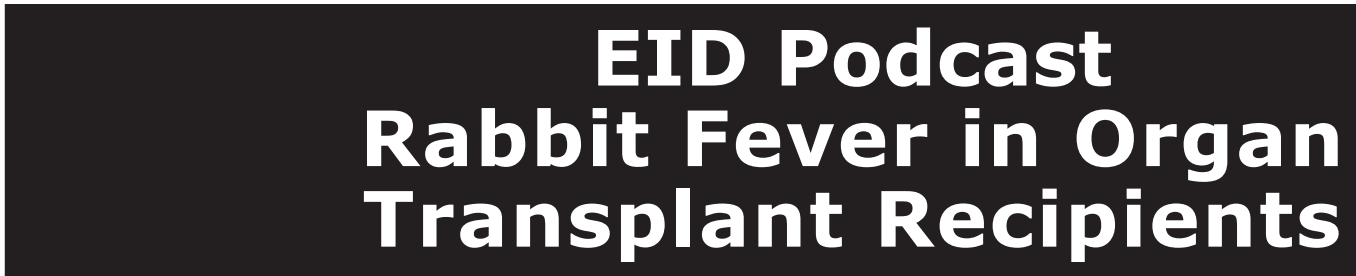

In July 2017, three people developed tularemia, or "rabbit fever," after receiving organ transplants from the same donor. Donated organs are routinely screened for common viruses, but unusual diseases like tularemia can sometimes go undetected.

In this April, 2019 EID podcast, Dr. Matthew Kuehnert, the medical director for the ation's largest tissue bank, MTF Biologics, explains how clinicians identified and diagnosed this rare disease.

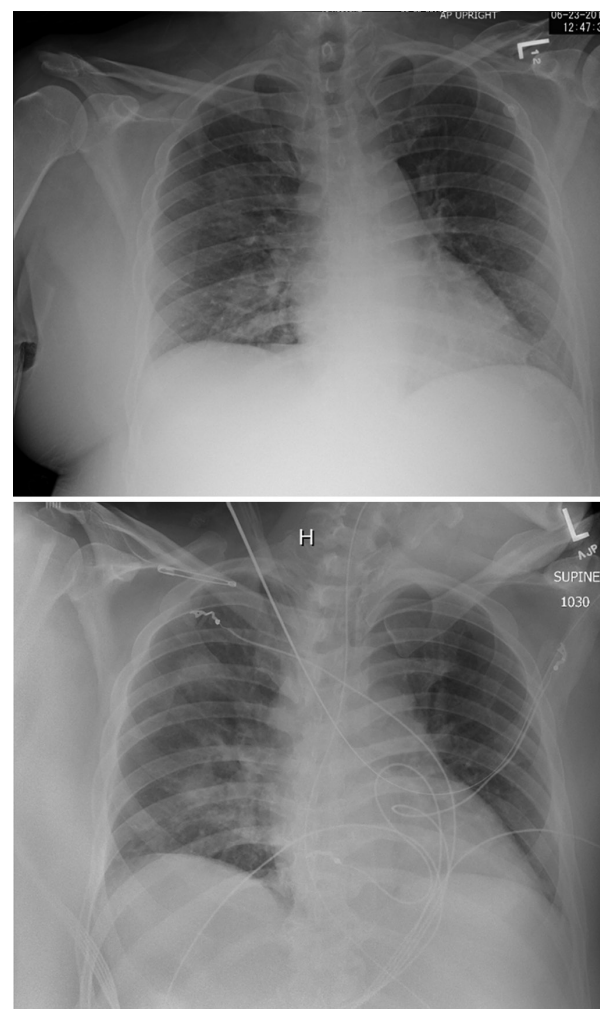

Visit our website to listen: EMERGING https://tools.cdc.gov/medialibrary/index.aspx\#/media/id/397813 INFECTIOUS DISEASES 